Int. J. Electrochem. Sci., 14 (2019) 1069 - 1081

\title{
Hydrothermal Synthesis of Manganese Sulfide Decorated Graphene Oxide for Effective Electrochemical Sensing of Dopamine
}

\author{
Settu Ramki ${ }^{1}$, Karuppaih Pandi ${ }^{1}$, Shen-Ming Chen ${ }^{* 1}$, Yi-Ting Ye ${ }^{1}$, Tse-Wei Chen ${ }^{1}$, Qingli Hao*2 \\ ${ }^{1}$ Electroanalysis and Bioelectrochemistry Lab, Department of Chemical Engineering and \\ Biotechnology, National Taipei University of Technology, Taipei 10608, Taiwan. \\ ${ }^{2}$ Key Laboratory for Soft Chemistry and Functional Materials, NanjingUniversity of Science and \\ Technology, Ministry of Education, Nanjing,China. \\ *E-mail: smchen78@ms15.hinet.net (Shen-Ming Chen); haoqingli@yahoo.com (Qingli Hao)
}

doi: $10.20964 / 2019.01 .58$

Received: 13 October 2018 / Accepted: 13 November 2018 / Published: 30 November 2018

\begin{abstract}
Microsphere like structured manganese sulfide decorated graphene oxide (MnS/GO) composites were synthesized by using simple hydrothermal method. The above prepared MnS/GO composite was characterized by using X-ray diffraction spectroscopy (XRD), Scanning Electron Microscopy (SEM), Energy Dispersive X-ray analysis (EDX), and Raman spectroscopy. The obtained results confirmed the formation of $\mathrm{MnS} / \mathrm{GO}$ composite with required stoichiometry and morphology. For the first time, $\mathrm{MnS}$ based materials were used as a modifier and dopamine as a model system for the electrochemical application. The electrochemical studies were performed for MnS/GO composite modified GCE using Cyclic Voltammetry (CV) and linear sweep voltammetry techniques. The results suggest that the effective response of MnS/GO/GCE with very low limit of detection (LOD) and sensitivity of $0.007 \mu \mathrm{M}$ and $3.11 \mu \mathrm{A} \mu \mathrm{M}^{-1} \mathrm{~cm}^{-2}$ respectively. Moreover the selectivity results exhibited excellent interference property of MnS/GO/GCE towards sensing of dopamine. Moreover, the reported MnS/GO/GCE sensor exhibits excellent long cyclic stability, and selectivity and greater support for the real-time detection of DA in human urine samples. Thus, the proposed MnS/GO/GCE is believed as an excellent electrode material for the sensing of dopamine.
\end{abstract}

Keywords: MnS microspheres, graphene oxide, dopamine, electrochemical sensor, LSV technique

\section{FULL TEXT}

(C) 2019 The Authors. Published by ESG (www.electrochemsci.org). This article is an open access article distributed under the terms and conditions of the Creative Commons Attribution license (http://creativecommons.org/licenses/by/4.0/). 